\title{
CARACTERIZACIÓN MORFOLÓGICA DE PELÍCULAS DELGADAS DE CU/CD OBTENIDAS POR ELECTRODEPOSICIÓN
}

Por:

William Aperador' ${ }^{1}$, Ariel Camargo', Cesar Ortiz' ${ }^{1}$ Enrique Vera' ${ }^{1}$.

\section{RESUMEN}

Este trabajo presenta el uso de la técnica de corriente directa y la implementación de las técnicas de corriente pulsante con inversión (PRC) y sin inversión de polaridad (PDC) para electrodepositar películas delgadas $\mathrm{Cu} / \mathrm{Cd}$ en forma de bicapa sobre substratos de Zamak. Se estudió la influencia de las diferentes variables involucradas en cada uno de los procesos: voltaje y tiempo de deposición (VHigh y tHigh), voltaje y tiempo de disolución (VLow y tlow) y el ciclo de

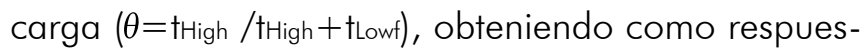
ta el monitoreo de la corriente anódica (Low) y catódica ( $\left.\right|_{\text {High }}$. Los electrodepósitos de $\mathrm{Cu} / \mathrm{Cd}$ se caracterizaron mediante microscopia óptica de barrido para hallar el tamaño de grano además, se halló la rugosidad utilizando Scanning Probe Image Processor (SPIP). Se observó que el control pulsante del voltaje durante el depósito permite obtener películas de Cu/Cd más densas y de tamaño de grano más fino, que las películas convencionales depositadas por la técnica de corriente directa. Además Para evaluar la potencia protectora anticorrosivas de las películas se utilizó la técnica TAFEL.

Palabras claves: Corriente Pulsante Inversa, corrosión, electrodeposición, películas delgadas.

\section{ABSTRACT}

This work presents the use of the technique of current pulsant inverse, pulse direct current and direct current for electrodeposition thin films $\mathrm{Cu} / \mathrm{Cd}$ in double-layer shape on Zamak substrate. The influence of the different variables was studied involved in each one of the processes: voltage and time of deposition ( V High and $V_{\text {Low }}$ ), voltage and time of breakup (VLow and tLow) and the load cycle $(\theta=$ tHigh/tHigh +tLow), obtaining the average of the anodic (lLow) and cathodic (IHigh) Currents as a function of the anodic (tow) and cathodic (tHigh) times, respectively. The electrodeposits of $\mathrm{Cu} / \mathrm{Cd}$ were characterized by means of optic microscopy to obtain the grain size; moreover the ruggedness was obtained using a Scanning Proved Image Processor (SPIP). These results showed that the control of pulsed voltage during the deposit allowing to obtain $\mathrm{Cu} / \mathrm{Cd}$ films denser, and with a size of grain finer that the conventional films deposited by the technique of direct current. The protective anticorrosive power of the thin films was evaluated by means the technique TAFEL.

Keywords: Corrosion, current pulsant inverse, electrodeposition, thin films.

\section{INTRODUCCIÓN}

Los procesos de electrodeposición de diferentes tipos de metales y aleaciones, son procesos que están encaminados a la consecución de características superficiales requeridas por diferentes tipos de materiales para diversas aplicaciones, como la protección frente a la acción de agentes corrosivos, el mejoramiento de su resistencia al desgaste y la obtención de superficies metálicas con acabados superficiales específicos [1]. En la actualidad, los procesos industriales de deposición electrolítica emplean la técnica de corriente directa (DC) para la obtención de los diferentes tipos de recubrimiento. Sin embargo, con esta técnica se obtienen en algunos casos, distribuciones de metal no uniformes generando depósitos no homogéneos y produciendo en algunos casos el efecto "hueso de perro" [2].

Como parte de las medidas adoptadas para corregir estos inconvenientes, en los últimos años se ha venido estudiando el efecto que tiene sobre las propiedades de los recubrimientos la variación en la forma de su-

'GRUPO DE SUPERFICIES ELECTROQUÍMICA Y CORROSIÓN. Escuela de Física, Facultad de Ciencias Universidad Pedagógica y Tecnológica de Colombia Av. Central del Norte, Tunja, Boyacá, Colombia Tels: 57 (8) 7422174-75. Ext. 1803, 1800

E-mail:grupo_gsec@yahoo.es 


\section{CARACTERIZACIÓN MORFOLÓGICA DE PELÍCULAS DELGADAS DE CU/CD OBTENIDAS POR \\ ELECTRODEPOSICIÓN}

ministrar la corriente directa, lo cual ha generado la aparición de las técnicas de corriente pulsante (PDC) y corriente pulsante inversa (PRC). La técnica PDC, es un proceso en el cual la corriente directa es aplicada por un periodo de tiempo especifico para que haya deposición (ton) y luego es llevada a un valor en el que ésta no ocurre durante otro lapso de tiempo, toff, (Fig. 1); en el caso de la técnica PRC se presenta un proceso de electrodeposición bipolar, en el cual la corriente directa está en constante cambio de polaridad (Fig. 2), en este caso, la electricidad que está fluyendo a través de los electrodos durante el período catódico, ton, debe ser mayor que la cantidad de electricidad que está fluyendo durante el tiempo del período anódico, trev, (Ec.1) [3]

$$
l_{c} t_{o n}>l_{a} t_{r e v}
$$

Figura 1. Esquema de la técnica PDC.

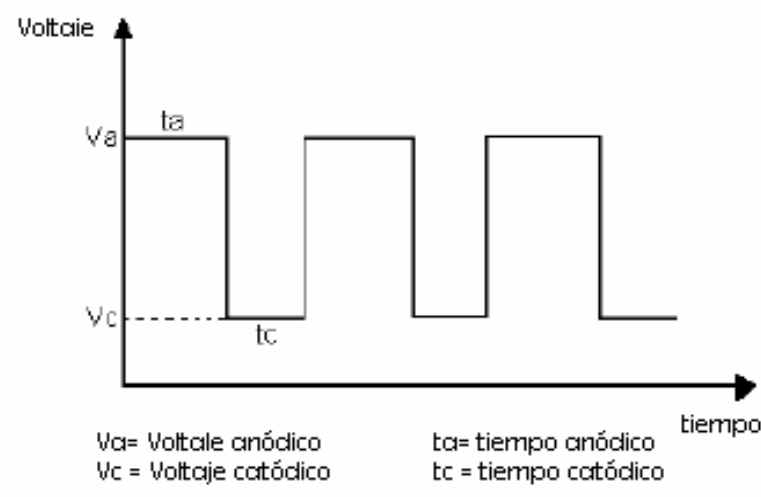

Figura 2. Esquema de la técnica PRC [2].

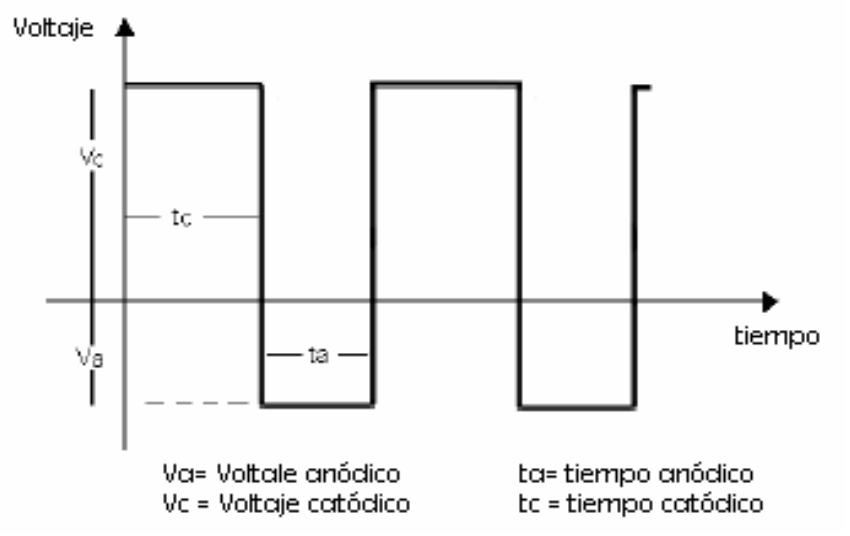

Por este método de deposición la cantidad de electricidad total no es consumida en la formación del depósito. Una porción de corriente significativamente más pequeña es utilizada para la polarización anódica de los productos recubiertos.

La aplicación de estas dos técnicas en la obtención de recubrimientos permite la variación de sus propiedades, ya que los parámetros de pulso involucrados afectan directamente los fenómenos de nucleación y crecimiento de los cristales que generan el depósito. Roy y Landolt (1997) [4] citaron para el caso de la técnica PRC cuatro parámetros de pulso a considerar: las densidades de corriente catódica y anódica, el período del pulso, $\mathrm{T}$, definido como la suma del ton y el trev y el ciclo de carga, $\theta$, definido como la relación entre el ton y el periodo $\left(\theta=t_{\text {on }} / T\right)$.

Por todo lo anterior, una de las metas de este trabajo fue obtener películas delgadas de $\mathrm{Cu} / \mathrm{Cd}$ utilizando las técnicas de corriente pulsante y corriente pulsante inversa. Para conocer la calidad de estas películas se les realizó una caracterización de rugosidad y tamaño de grano. Los resultados mostraron que cuando se utilizan dichas técnicas, se obtienen películas de mejor calidad que las obtenidas por el método tradicional de corriente directa.

\section{DESARROLLO EXPERIMENTAL}

Los recubrimientos de cobre fueron depositados sobre discos de $15 \mathrm{~mm}$ de Zamak la cual es una aleación que se fabrica con Zinc de $99.99+\%$ de pureza, Aluminio y Magnesio puros y Cobre electrolítico. Se empleo un electrolito alcalino cuya composición y condiciones de trabajo se encuentran descritas en la Tabla 1. Los recubrimientos de cadmio fueron electrodepositados sobre los cátodos de zamak recubiertos con cobre, en la Tabla 1 se citan las condiciones de operación y las características del electrolito empleado.

Todos los electrorecubrimientos se obtuvieron empleando un hardware (rectificador de corriente) de 30 amperios controlado mediante un software diseñado en Labview, el cual permite la variación de los diferen- 


\section{CARACTERIZACIÓN MORFOLÓGICA DE PELÍCULAS DELGADAS DE CU/CD OBTENIDAS POR ELECTRODEPOSICIÓN}

tes parámetros de pulso involucrados en el proceso, tales como el voltaje catódico, el voltaje anódico, el voltaje de relajación, y los tiempos catódico, de relajación y de disolución del proceso.

Tabla 1. Características de los electrolitos de cobre y cadmio

\begin{tabular}{|c|c|c|c|}
\hline \multicolumn{2}{|c|}{ Electrolito de Cadmio } & \multicolumn{2}{c|}{ Electrolito de Cobre } \\
\hline \hline Parámetro & Valor & Parámetro & Valor \\
\hline \hline $\mathrm{NaCN}$ & $56 \mathrm{~g} / \mathrm{l}$ & $\mathrm{CdO}$ & $27 \mathrm{~g} / \mathrm{l}$ \\
\hline $\mathrm{CuCN}$ & $42 \mathrm{~g} / \mathrm{l}$ & $\mathrm{NaCN}$ & $120 \mathrm{~g} / \mathrm{l}$ \\
\hline \hline $\mathrm{KNaH}{ }_{4} \mathrm{O}_{6} 4 \mathrm{H}_{2} \mathrm{O}$ & $45 \%$ & $\mathrm{Na}_{2} \mathrm{SO}_{4}$ & $50 \mathrm{~g} / \mathrm{l}$ \\
\hline \hline Ánodos $(\% \mathrm{Cu})$ & $99.9 \%$ & Ánodos $\% \mathrm{Cu})$ & $99.5 \%$ \\
\hline \hline $\mathrm{pH}$ & 11 & $\mathrm{pH}$ & 4.2 \\
\hline \hline Temperatura & $35^{\circ} \mathrm{C}$ & Temperatura & $40{ }^{\circ} \mathrm{C}$ \\
\hline \hline Tiempo de proceso & $45 \mathrm{~min}$ & Tiempo de proceso & $75 \mathrm{~min}$ \\
\hline
\end{tabular}

El esquema del software que se desarrollo es el mostrado en la figura 3 en donde se presentan las tres técnicas que se utilizaron para obtener los recubrimientos. La técnica $D C$ es la que se observa en la parte izquierda, la PDC la observamos en la parte derecha y por ultimo la técnica PRC en la parte inferior. Los parámetros empleados en cada uno de los ensayos para la obtención de los recubrimientos de cobre y de cadmio con cada una de las técnicas empleadas se encuentran en las tabla 2.

Figura 3. Esquema del software desarrollado para la obtención de las electrodeposiciones con las técnicas DC, PDC, PRC.

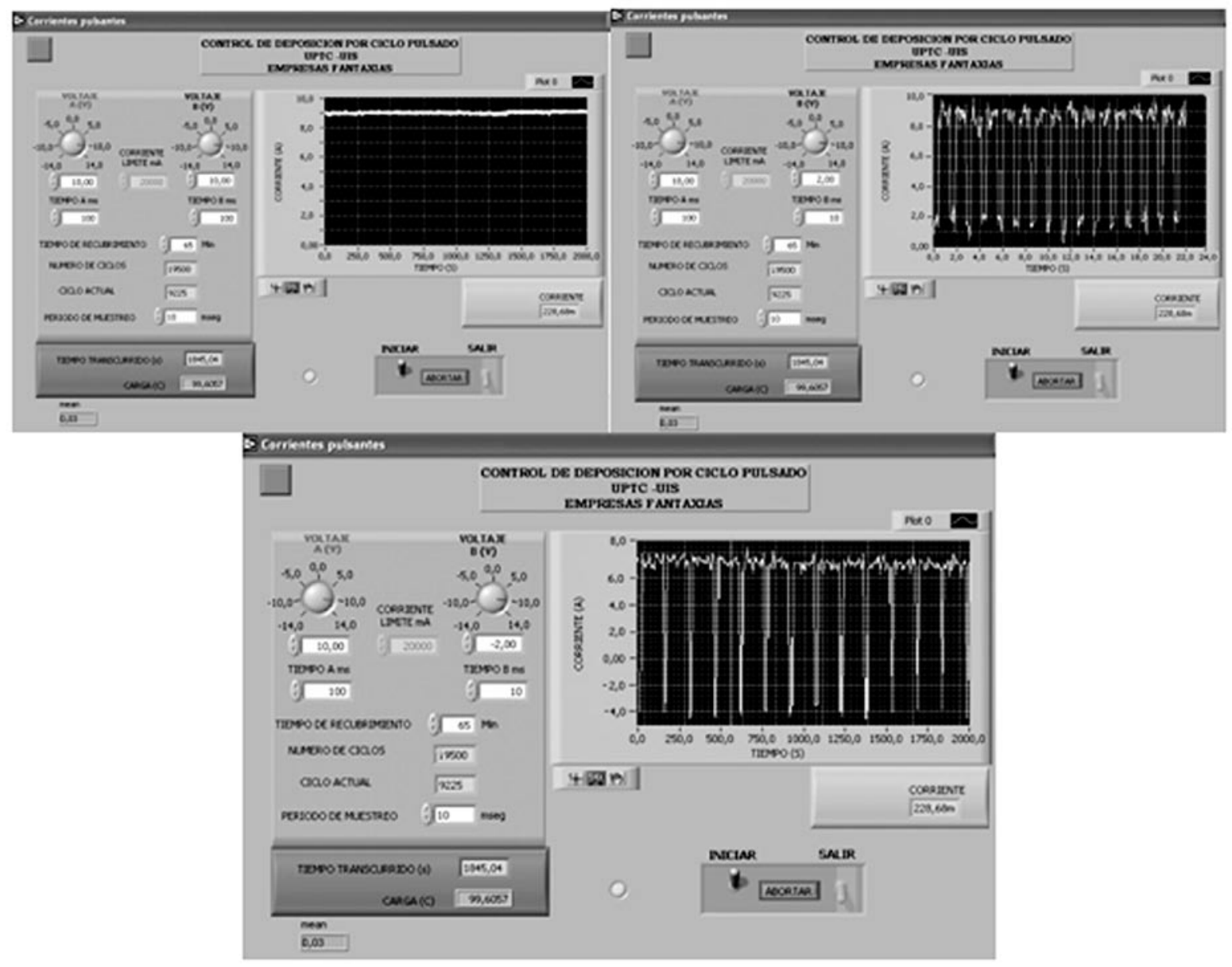




\section{CARACTERIZACIÓN MORFOLÓGICA DE PELÍCULAS DELGADAS DE CU/CD OBTENIDAS POR \\ ELECTRODEPOSICIÓN}

Tabla 2. Parámetros utilizados en la obtención de electrorecubrimientos de cadmio.

\begin{tabular}{|c|c|c|c|c|c|c|}
\hline \multicolumn{2}{|l|}{ Técnica } & $\begin{array}{l}V_{\text {on }} \\
(V)\end{array}$ & $\begin{array}{l}V_{\text {of }} \\
\text { (V) }\end{array}$ & $\begin{array}{l}V_{\text {rev }} \\
\text { (V) }\end{array}$ & $\begin{array}{c}T_{\text {on }} \\
(\mathrm{ms})\end{array}$ & $\begin{array}{c}T_{\text {off }} \\
(\mathrm{ms})\end{array}$ \\
\hline \multirow[b]{2}{*}{ DC } & 7 & 10 & - & - & - & - \\
\hline & 2 & 8 & 2 & - & - & - \\
\hline \multirow[b]{2}{*}{ PDC } & 1 & 10 & 2 & - & 100 & 10 \\
\hline & 2 & 8 & 2 & - & 100 & 10 \\
\hline \multirow{2}{*}{ PRC } & 1 & 10 & - & -2 & 100 & 10 \\
\hline & 2 & 8 & - & -4 & 100 & 10 \\
\hline
\end{tabular}

Antes del proceso de cobrizado, los cátodos fueron sometidos a un proceso de limpieza en ultrasonido sumergidos en una solución de compuesta de $\mathrm{CH} 3 \mathrm{COCH} 3$. El equipo utilizado para el ultrasonido es un Elma D-78224 el cual entrega una frecuencia HF $35 \mathrm{kHz}$. Luego del ultrasonido se realizó un desengrase electrolítico hecho con un jabón industrial llamado Z-120 el cual básicamente es alquilarilsufonatos, luego se hizo un decapado en ácido sulfúrico al $5 \%$ en volumen.

La determinación del tamaño de grano y el análisis superficial de los depósitos se llevó a cabo empleando un Microscopio de Barrido por Sonda en el modo de Microscopía de Fuerza Atómica en Contacto (AFM) el equipo utilizado es un auto probe $\mathrm{cp}$ park de la firma scientific instruments. La medida de rugosidad se obtuvo con el programa ProScan Image Procesing para un área de $10 \mu \mathrm{m} 2$. La caracterización electroquímica se realizó en un equipo Gamry modelo PC-4 mediante la medida de curvas de polarización (Tafel), a temperatura ambiente, empleando una celda compuesta por el electrodo de trabajo con un área expuesta de $1 \mathrm{~cm} 2$, un electrodo de referencia de $\mathrm{Ag} / \mathrm{AgCl}$ y un alambre de platino como contra electrodo en una solución de $\mathrm{HCl}$ al $1 \%$ preparada con agua destilada, se eligió esta solución debido a que se utiliza para limpiar metales en las industrias de galvanoplastia además corroe metales activos formando los cloruros del metal e hidrógeno. Los diagramas de Tafel se obtuvieron a una velocidad de barrido de $0.5 \mathrm{mV} / \mathrm{s}$ en un rango de voltajes de $-0.25 \mathrm{~V}$ a $1 \mathrm{~V}$ empleando un área expuesta de $1 \mathrm{~cm} 2$. Las pruebas de microdureza se realizaron con un microdurometro digital modelo HVS 1000, con una presión de prueba de $2.94 \mathrm{~N}$.

\section{RESULTADOS Y DISCUSIÓN}

\subsection{TAMAÑO DE GRANO DE LOS RECUBRI- MIENTOS DE CADMIO}

Figura 4. Topografías de los electrorecubrimientos de cadmio obtenidas con las técnicas (a) DC; (b) PDC; (c) PRC.

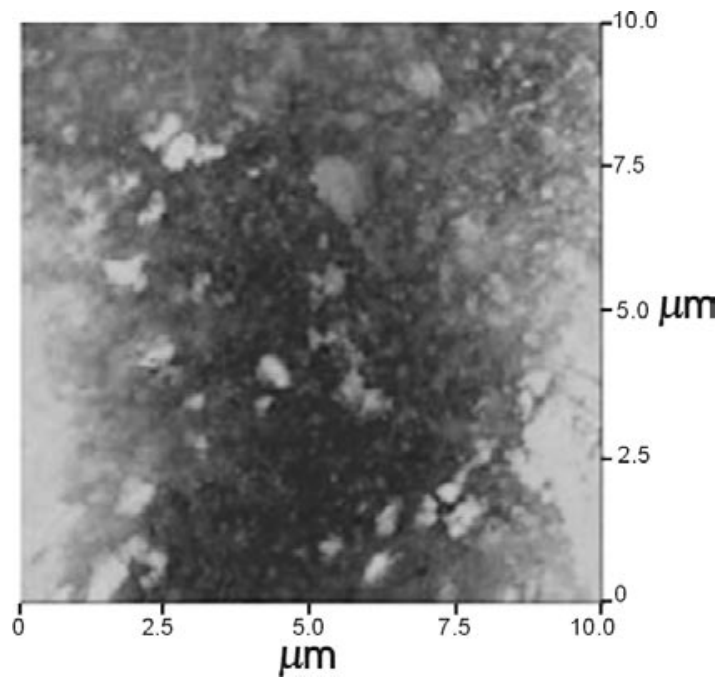

(a)

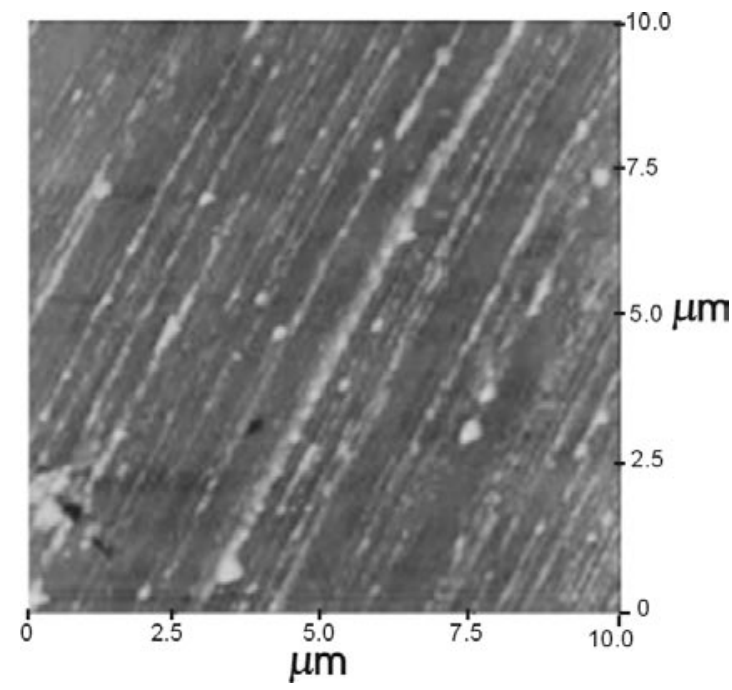

(b) 


\section{CARACTERIZACIÓN MORFOLÓGICA DE PELÍCULAS DELGADAS DE CU/CD OBTENIDAS POR \\ ELECTRODEPOSICIÓN}

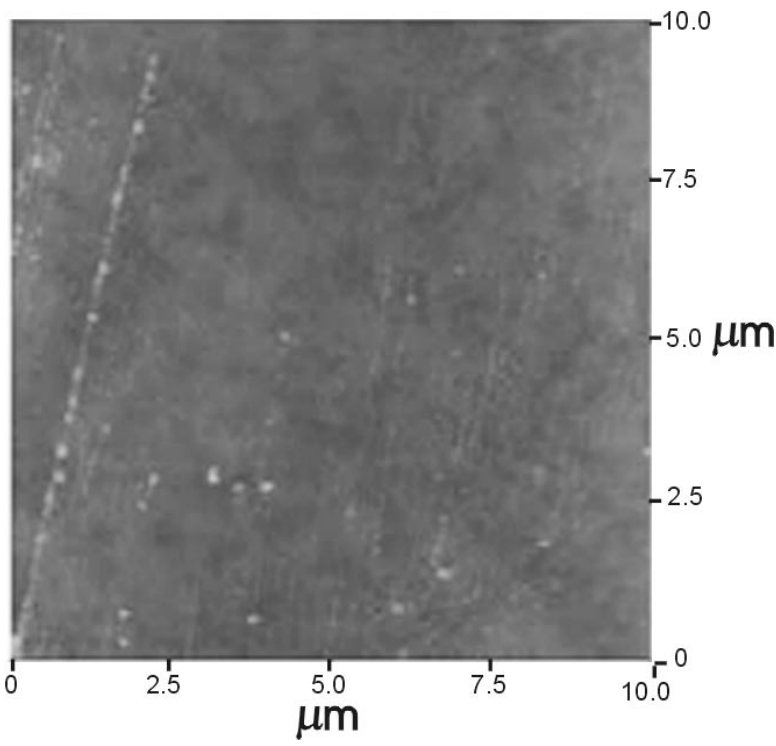

(c)

En la Fig. 4 se observan las diferentes topografías de los recubrimientos de cadmio electrodepositados con las técnicas DC (Fig. 4a), PDC (Fig. 4b) y PRC (Fig. 4c). Es claramente observable que los depósitos obtenidos con corriente pulsante inversa presentan un tamaño de grano muy fino comparado con las otras dos técnicas y a su vez una distribución más homogénea. En el caso de los recubrimientos obtenidos con corriente directa se encontró un tamaño de grano de $4.25 \mu \mathrm{m}$, mientras que con corriente pulsante y corriente pulsante inversa se obtuvieron en promedio un tamaño de grano de 1.652 y $1.298 \mu \mathrm{m}$, respectivamente.

Estos resultados concuerdan con los obtenidos por Mishra y Balasubramaniam[5], donde se evidencia que el empleo las técnicas de corriente pulsante y corriente pulsante inversa inducen velocidades más altas de nucleación de los cristales, por lo cual se obtienen estructuras con tamaño de grano más refinado. La diferencia de color en la figura 3 a se debe a los diferentes tamaños de grano que se encuentran en esta probeta.

\subsection{MICRODUREZA DE LOS RECUBRIMIENTOS DE CADMIO}

Las mediciones de microdureza realizadas en los recubrimientos de cadmio se muestran en la Tabla 3. Los resultados obtenidos permiten ver el efecto positivo de las técnicas de corriente pulsante sobre el valor de la dureza de este tipo de recubrimientos. En ambos casos se encontró un aumento en la microdureza, que probablemente guarda estrecha relación con el tamaño de grano más fino presente en estos tipos de depósitos. [6]

Tabla 3. Valores promedio de la microdureza vickers para las probetas de $\mathrm{Cu} / \mathrm{Cd}$.

\begin{tabular}{|c|c|}
\hline Técnica & Microdureza vickers (Valor Promedio) \\
\hline DC & 64 \\
\hline PDC & 152.5 \\
\hline PRC & 356 \\
\hline
\end{tabular}

\subsection{CURVAS DE POLARIZACIÓN}

En la Figura 5 se muestran los diagramas de Tafel correspondientes a los depósitos de cadmio que se obtuvieron empleando las técnicas DC, PDC y PRC.

Figura 5. Diagramas de Tafel correspondientes a los depósitos de cadmio que se obtuvieron empleando las técnicas DC, PC y PRC.

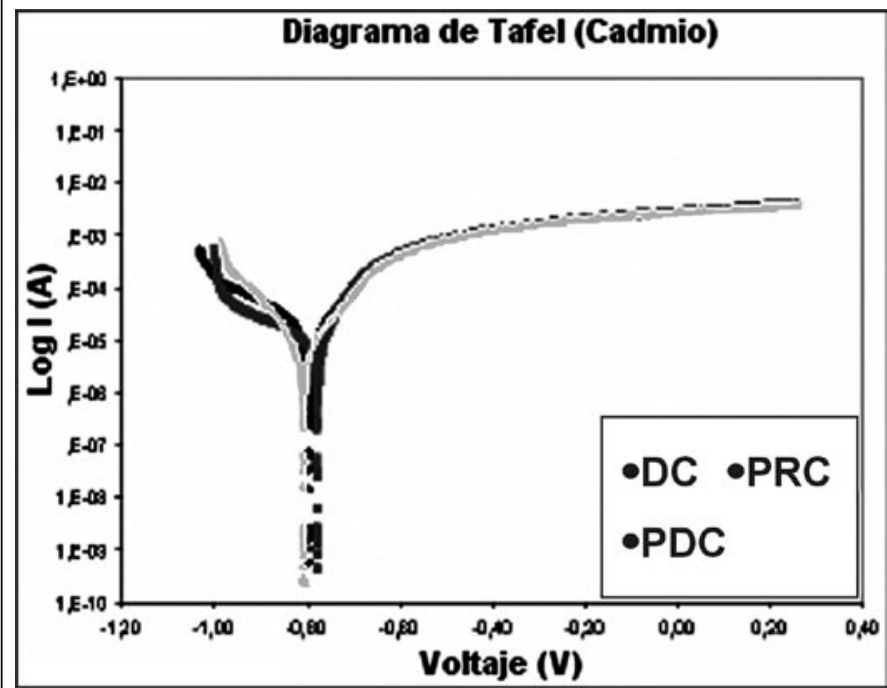




\section{CARACTERIZACIÓN MORFOLÓGICA DE PELÍCULAS DELGADAS DE CU/CD OBTENIDAS POR \\ ELECTRODEPOSICIÓN}

Las curvas de polarización obtenidas permiten encontrar los valores de las pendientes anódica y catódica en cada caso [7], los cuales se muestran en la tabla 4 junto con los valores de densidad de corriente y velocidad de corrosión para cada uno de los casos estudiados, las curvas de polarización que se halaron muestra que el comportamiento de las 3 técnicas es similar la diferencia de una frente a otra radica en las pendiente que se hallaron las cuales modifican la corriente de corrosión y a su vez también modifica la velocidad de la corrosión pero en valores muy pequeños tal como se muestra en la tabla 4.

Tabla 4. Valores de los parámetros utilizados para hallar la velocidad de degradación del cadmio.

\begin{tabular}{|c|c|c|c|}
\hline Parámetro & Técnica DC & Técnica PDC & Técnica PRC \\
\hline $\begin{array}{c}\text { Beta Anódico } \\
(V \text { década) }\end{array}$ & 0.1419 & 0.1015 & 0.0986 \\
\hline $\begin{array}{c}\text { Beta Catódico } \\
(V \text { década) }\end{array}$ & 0.2401 & 0.2480 & 0.0935 \\
\hline $\begin{array}{c}\text { Corriente de } \\
\text { Corrosión (A) }\end{array}$ & $2.16 e^{-6}$ & $9.65 e^{-6}$ & $5.65 e^{-6}$ \\
\hline $\begin{array}{c}\text { Velocidad de } \\
\text { Corrosión (mpy) }\end{array}$ & 36.1359 & 16.1431 & 9.4564 \\
\hline
\end{tabular}

Los parámetros encontrados con ayuda de las curvas de polarización permiten hacer uso de la ecuación de Stern - Geary [2] para hallar las densidades de corriente de corrosión. Se determinó que la velocidad de corrosión más alta se hallaba en los electrodepositos obtenidos con corriente directa a un voltaje de $10 \mathrm{~V}$, encontrándose un valor de 36.1359 mpy, dato que muestra concordancia con los resultados obtenidos durante la evaluación de topografía en donde se hallaron depósitos menos uniformes y más rugosos con esta técnica. En el caso de la corriente pulsante las condiciones de deposición empleadas no dejan ver un cambio significativo en el comportamiento frente a la corrosión de los depósitos, obteniéndose valores intermedios de 16.1431 mpy. Con la técnica de corriente pulsante inversa no se notó una influencia marcada del voltaje anódico en el grado de deterioro del depósito, sin embargo, el aumento del tiempo de pulso anódico genera un depósito con un tamaño de grano más refinado, con un mayor grado de compactación y con una mejor respuesta frente al ataque corrosivo estudiado, encontrándose valores de velocidades de corrosión muy reducidos 9.4564 mpy, en comparación con las otras dos técnicas evaluadas.

\section{CONCLUSIONES}

1. El empleo de las técnicas de corriente pulsante con y sin inversión de polaridad, resulta benéfico para la reducción del tamaño de grano de los recubrimientos de cadmio, ya que se ven directamente afectados los fenómenos de nucleación y crecimiento de los cristales.

2. Se encontró que las técnicas de corriente pulsante y corriente pulsante inversa, tienen influencia sobre la microdureza de los recubrimientos de cadmio. El empleo de una $u$ otra técnica contribuye a valores de dureza mayores en comparación con la técnica de corriente directa.

3. Con las curvas de polarización se obtuvo que los depósitos de cobre - cadmio, obtenidos con la técnica PRC son los que presentan menor velocidad de corrosión en comparación con las técnicas DC y PDC con parámetros de voltaje y tiempo catódico altos y voltaje y tiempo anódico bajo.

\section{REFERENCIAS}

[1]. LOWENHWEIM, F. 1963. Modern Electroplating. John Wiley \& Sons, Segunda edición, New York.

[2]. DUVA, R. 1996. Electroplating Engineering Handbook. Chapman y Hall, pp 684-690.

[3]. MANDICH, N. Pulse and pulse-reverse electroplanting. HBM Engineering Co, Lansing III. 
[4]. ROY, S. and LANDOLT, D. 1997. Determination of the practical range of parameters during reversepulse current plating, J. Appel Electrochem, Vol 27, pp. 299-307.

[5]. MISHRA, R. and BALASUBRAMANIAM, R. 2004. Effect of nanocrystalline grain size on the electrochemical and corrosion behavior of nickel. Corrosion Science, Vol 46, pp 3019.

[6]. LANDOLT, D. and MARLOT, A. 2003. Microstructure and composition of pulse-plated metals and alloys. Surface and Coatings Technology. p. $169-170$.

[7]. BARD, A. and FAULKNER, L. 2001. Electrochemical methods fundamentals and applications. John Wiley \& Sons, Second edition, New York.

Recibido: 29 - Octubre - 2006

Aceptado: 30 - Mayo - 2007 